\title{
Unsedated peroral percutaneous endoscopic gastrostomy (PEG): a safe and effective method
}

\author{
H. Steed, D. Barrett and S. D. Hearing \\ Gastroenterology Department, Stafford Hospital, Stafford, UK
}

Percutaneous endoscopic gastrostomy (PEG) insertion has traditionally been considered as an uncomfortable procedure requiring sedation. PEG insertion in some patients is not possible due to patient co-morbidity (e.g. respiratory) precluding safe sedation. In some units, it is possible to place radiologically inserted gastrostomy (RIG), but this option is not available in many hospitals. We present a case series of ten patients who have undergone unsedated peroral PEG insertion in the last three years in our hospital.

Patients were given 10 sprays of lidocaine $1 \%$ pharyngeal anaesthesia. The procedure was performed using the standard technique with a $9.2 \mathrm{~mm}$-diameter endoscope and local anaesthesia.

Eight of the ten patients were male with a mean age of 69 years old. In the majority of the patients the indication for pharyngeal anaesthesia was hypoxia due to respiratory co-morbidity and in two cases it was patient choice. Of the patients $(n 5)$ able to respond to questioning over the acceptability of the procedure patients responded that although it was uncomfortable they would be willing to have a PEG inserted using this method again. Where patients are unable to comment for themselves, the nurses observations are recorded all of which described the patient as calm and settled throughout the procedure. There were no endoscopy-related complications seen in this group, oxygen saturations remained at acceptable levels given their pre-procedure oxygen levels and for those patients who were able to be discharged from the endoscopy department were able to do so without the usual need for 24-hsupervision. Two patients suffered from re-feeding syndrome requiring electrolyte correction. Thirty day mortality was $30 \%$ with no cases attributed to the endoscopic procedure.

In a selected group of patients unsedated PEG insertion is safe, well tolerated and acceptable to patients. These data demonstrate that the technique of unsedated PEG placement should be considered in patients who are at high risk from sedation and that this may reduce the delay in PEG placement and the commencement of enteral nutrition. 